\title{
Characterization of Carotid Atherosclerotic Plaques by Measurement of Acoustic Impedance
}

\author{
Bükem Bilen ${ }^{1 *}$, Ülkü Kafa ${ }^{2}$, Mehmet Burçin Ünlü ${ }^{1}$ \\ ${ }^{1}$ Department of Physics, Faculty of Science and Arts, Boğaziçi University, 34342, Istanbul, Turkey. \\ ${ }^{2}$ Department of Cardiovascular Surgery, Mehmet Akif Ersoy Chest, Heart and Vascular Surgery Education and \\ Research Hospital, 34303, Istanbul, Turkey. \\ *bukem.bilen1@boun.edu.tr
}

Received: 11 July 2018

Accepted: 17 September 2018

DOI: $10.18466 /$ cbayarfbe. 442817

\begin{abstract}
In this study, acoustic properties of carotid atherosclerotic plaques are studied ex vivo by scanning acoustic microscope (SAM). Human carotid atherosclerotic plaques were collected from patients by carotid erdarterectomy operation technique. An $80 \mathrm{MHz}$ transducer was used in SAM, during the observation of the plaques. For the fibrous tissue, acoustic impedance was measured as $2.02 \pm 0.06$ MRayl, while for the lipid pool it was measured as $1.70 \pm 0.07$ MRayl and for the calcified region within the intima it was measured as $2.23 \pm 0.09$ MRayl. The difference in acoustic impedance values is due to the variation of elasticity within the atherosclerotic plaques. Lipid pool, together with fibrous tissue and calcified regions are the indications of vulnerable plaques, therefore, the determination of these regions within the plaques by SAM will help to evaluate the risk of thrombosis or stenosis and effect the decision making of the operations of the patients.
\end{abstract}

Keywords: Scanning acoustic microscopy, carotid artery, atherosclerosis, vulnerable plaque, acoustic impedance.

\section{Introduction}

Vulnerable atherosclerotic plaques, which can start developing even at childhood, have to be treated immediately by practitioners, since the rupture of the plaques may be fatal. The components within the plaques are the indications of the plaque type, therefore, the characterization of these components is widely studied. The accumulation of lipids and calcium under a thin fibrous cap makeup the so-called vulnerable plaques $[1,2]$ and the rupture of this thin cap will cause thrombosis or stenosis. The identification of the morphology of the arteries together with the chemical composition of the plaques are crucial for locating a vulnerable plaque and therefore, for the treatment of the patients.

The technologies for tissue imaging in the vessel wall are limited but in progress. Structural information of the atherosclerotic plaques can be obtained by intravascular ultrasound (IVUS) with an axial resolution of 100-200 $\mu \mathrm{m}[3,4]$. IVUS is today a widely used method in clinics. Another intravascular imaging method, optical coherence tomography (OCT), has a lower penetration depth but a better axial resolution of $10 \mu \mathrm{m}$ [5]. Even though flushing of the artery is necessary due to strong signal attenuation through blood, compared to IVUS, OCT offers better assessment of the atherosclerotic plaques. However, these two techniques provide only morphological assessment of the vessel. In intravascular photoacoustic (IVPA) imaging, laser pulses of several nanoseconds are absorbed by the tissue under investigation and absorption of the laser light gives rise to an acoustic wave. This acoustic wave is detected by an ultrasonic transducer. Two primary advantages of IVPA imaging are resolution and viewing depth of ultrasound and chemical specificity of optical absorption [6]. Combining two or more imaging methods provides complementary information for the detection of tissue abnormalities [7, 8]. Acoustic imaging, which is another recent diagnostic technique, can discriminate between different tissue types. The observation of the biological tissues by acoustic microscope can be done quickly without requiring staining. SAM obtains the image of an area of around 5 $\mathrm{mm} \times 5 \mathrm{~mm}$ in a couple of minutes. The elastic parameters of the tissues can be determined at microscopic level. Its combination with photoacoustic microscopy [9] can further be studied.

In this study, the acoustic properties of carotid atherosclerotic plaques were determined ex vivo by SAM. The measurement of acoustic impedance values will help determining the plaque components and therefore assessing the vulnerability of the plaques. 


\section{Materials and Methods}

\subsection{Tissue Preparation}

Carotid atherosclerotic plaques were received from patients, whose consent were taken, undergoing carotid endarterectomy operation, as ethically approved study by Istanbul Mehmet Akif Ersoy Chest, Heart and Vascular Surgery Education and Research Hospital Ethics Committee (Number: 2017-18). The incision is made parallel to the stenocleidomastoid muscle's anterior border. After intravenous heparinisation (5000 units), a longitudinal arteriotomy is made. The insicion is extended into the internal carotid artery. A temporary carotid balloons shunt is inserted for the cerebral circulation. A dissector is used to remove the plaque from the adventitia. After removing the plaque, arteriotomy is repaired using a prosthatic patch with $6 / 0$ prolene suture.

The plaques, stored in sterile saline, were received within 3-4 h post-surgery and immediately observed by SAM. The plaques had cylinder-like structures of a height of around $2 \mathrm{~cm}$ and a radius of approximately 1 $\mathrm{cm}$. By slicing them with a lancet, we prepared smaller cross sections of a height of $2 \mathrm{~mm}$ with plain facets, for SAM studies and then placed them on a polystyrene substrate, as shown in Figure 1.

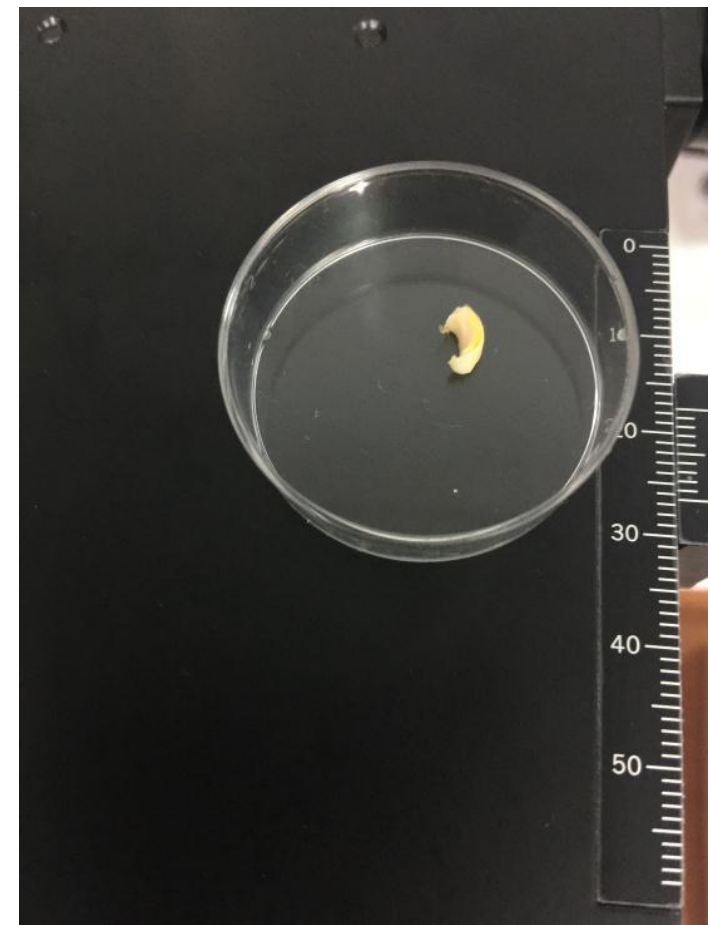

Figure 1. Cross-sectional view of an atherosclerotic plaque on a polystyrene substrate. The experiments are performed on cross-sections to obtain elasticity variations of different components within the atherosclerotic plaques.

\subsection{Experimental Setup}

Scanning acoustic microscope, AMS-50SI, developed by Honda Electronics (Toyohashi, Japan), was used to image two-dimensional distribution of the acoustic intensity and impedance of the atherosclerotic plaques.

The diagram for the acoustic impedance microscopy is shown in Figure 2. SAM is mainly composed of five units: (1) Transducer with acoustic lens, (2) pulser/receiver, (3) oscilloscope, (4) stage control computer and (5) display monitor. The transducer was generating and receiving a single pulse of width of $5 \mathrm{~ns}$ with a repetition rate of $10 \mathrm{kHz}$. The central frequency of the transducer and its bandwidth were $80 \mathrm{MHz}$ and 50-105 MHz, respectively. The transducer with sapphire lens was fixed on a stage, which was controlled by the computer. The transducer detect the reflections from both the reference and target material, then these reflections were analyzed by the oscilloscope. As a result, two-dimensional distributions of acoustic intensity and impedance of the region of interest of maximum $4.8 \mathrm{~mm} \times 4.8 \mathrm{~mm}$ or minimum $0.3 \mathrm{~mm} \times 0.3$ $\mathrm{mm}$ were visualized with $300 \times 300$ pixels with a lateral resolution of approximately $20 \mu \mathrm{m}$.

The reference substance and the medium, which couples the substrate and the transducer, were distilled water.

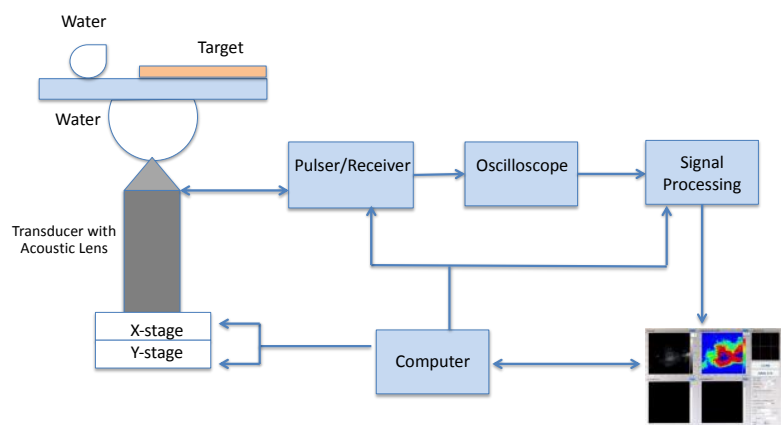

Figure 2. Diagram for the acoustic impedance microscopy. SAM has five units: (1) Transducer with acoustic lens, (2) pulser/receiver, (3) oscilloscope, (4) stage control computer and (5) display monitor.

\subsection{Principal of Acoustic Microscopy}

Figure 3 illustrates the schematic for the principle of acoustic impedance measurement by scanning acoustic microscope. The same transducer radiates and receives the acoustic wave. The target signal $\boldsymbol{S}_{\text {target }}$ is described as

$$
S_{\text {target }}=\frac{Z_{\text {target }}-Z_{\text {sub }}}{Z_{\text {target }}+Z_{\text {sub }}} S_{0}
$$

where $S_{0}$ is defined as the signal transmitted by the transducer, $Z_{\text {target }}$ is defined as the target's acoustic impedance and $Z_{\text {sub }}$ is defined as the substrate's acoustic impedance (2.37 MRayl). The reflected signal 
from the target is compared with the reflected signal from the reference and given as

$$
S_{\text {ref }}=\frac{Z_{\text {ref }}-Z_{\text {sub }}}{Z_{\text {ref }}+Z_{\text {sub }}} S_{0}
$$

where $Z_{\text {ref }}$ is defined to be the reference material's acoustic impedance (1.50 MRayl). The target's acoustic impedance is subsequently calculated as

$$
\begin{aligned}
Z_{\text {target }} & =\frac{1+\frac{S_{\text {target }}}{S_{0}}}{1-\frac{S_{\text {target }}}{S_{0}}} Z_{\text {sub }} \\
& =\frac{1-\frac{s_{\text {target }}}{S_{\text {ref }}}\left[\frac{Z_{\text {sub }}-Z_{\text {ref }}}{Z_{\text {sub }}+Z_{\text {ref }}}\right]}{1+\frac{S_{\text {target }}}{S_{\text {ref }}}\left[\frac{Z_{\text {sub }} Z_{\text {ref }}}{Z_{\text {sub }}+Z_{\text {ref }}}\right]} Z_{\text {sub }}
\end{aligned}
$$

where $S_{0}$ is assumed to be constant during the observation process [10, 11].
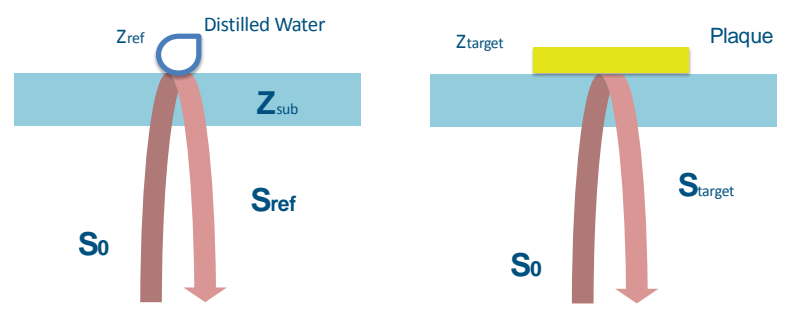

Figure 3. Schematic for the principle of acoustic impedance measurement by SAM. Reflected ultrasound signals from surfaces of water and the plaque sample are collected and compared to obtain acoustic impedance of the plaque.

\section{Results and Discussion}

The atherosclerotic plaques were sliced approximately 2 mm thick to obtain cross-sectional views, which were necessary for the comparison of different components within the plaques. PC window of the acoustic impedance microscope is shown in Figure 4. There are two images obtained in the acoustic mode, which are the intensity and acoustic impedance distribution images. The image on the left is the intensity image and the one on the right is the impedance distribution image, which are both constructed by the reflections from both surfaces of the reference and the target using the equations given in Section 2.3. The acoustic impedance distribution indicates different acoustic properties due to variation of elasticity within the atherosclerotic plaques. The impedance was determined to be $2.02 \pm 0.06$ MRayl for the fibrous tissue, 1.70 \pm 0.07 MRayl for the lipid pool and 2.23 \pm 0.09 MRayl for the calcified lesion in the intima. The calcific region was so stiff that it could have been distinguished easily from others. Similarly, fibrous tissue was stiffer than lipid, therefore, 3 regions were obviously separable. Each acoustic impedance value was the average obtained from multiple points within each area.

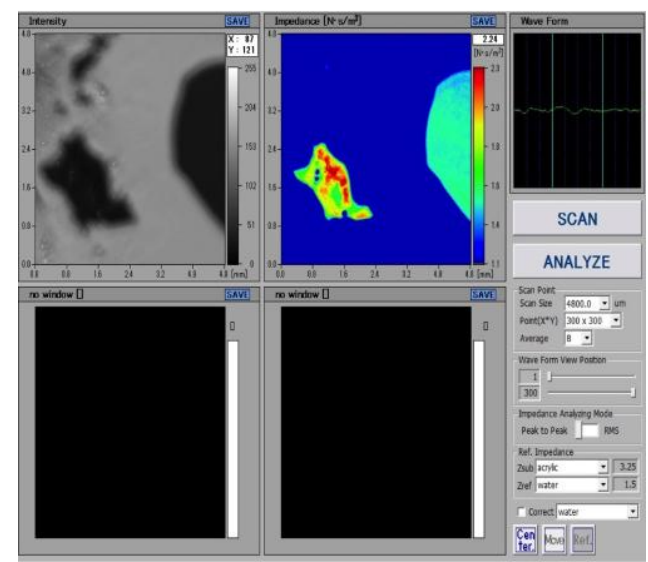

Figure 4. PC window of SAM showing cross-sectional view of an atherosclerotic plaque. Left top image is the intensity map and right top image is the acoustic impedance map of the sample. Scan area is $4.8 \times 4.8$ $\mathrm{mm}^{2}$.

The acoustic impedance microscopy image of the intima of an atherosclerotic plaque sample is shown in Figure 5. Calcified and fibrous plaques can be identified easily in IVUS imaging, with high intensity echo due to high scattering within these regions [12]. Similarly, the intensity image of acoustic impedance microscopy clearly shows the calcified region at the lower right corner. In the impedance image on the right, a representative point was chosen to show that the impedance value of the calcified lesion is greater than the impedance values of the lipid deposition and the fibrous tissue.

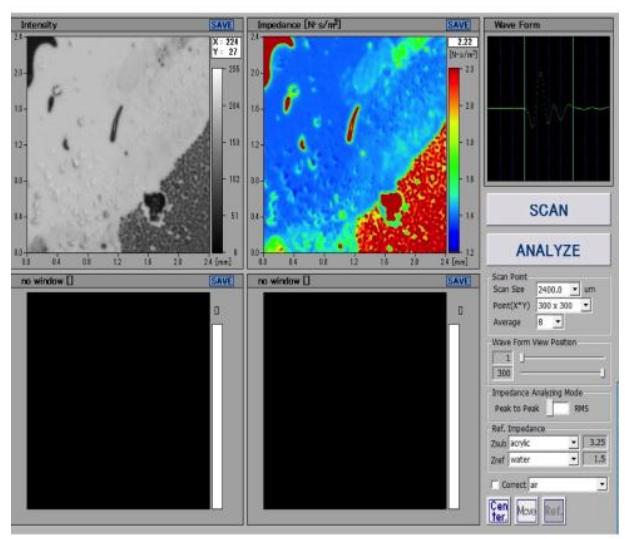

Figure 5. Acoustic impedance map of the intima of an atherosclerotic plaque sample. Left top image is the intensity map and right top image is the acoustic impedance map of the sample. Scan area is $2.4 \times 2.4$ $\mathrm{mm}^{2}$.

The degree of lipid deposition is critical for early stage diagnosis of atherosclerosis [2]. Fibrous cap over lipid- 
rich necrotic cores and just under endothelium is observed through the natural development of fibroatheroma [1]. This fibrous cap may become thin and weakened and is susceptible to rupture, then this lesion is labeled as vulnerable plaque. Plaques may Thin-cap fibroatheromas (TCFAs), which are fibroatheromas with a fibrous cap thickness of $65 \mu \mathrm{m}$ [13], are found to be at high risk for rupture. TCFA has a necrotic core and also calcium deposition [1, 2]. Calcification is considered to be an active process, which involves information about vulnerability [14]. In fact, microcalcification or spotty calcification within the plaque is found to be the precursor of cardiovascular complications [15]. Plaques with millimeter-sized or larger calcifications are said to be no longer inflammatory and stable $[16,17]$. Most of the carotid atherosclerotic plaques received from patients by carotid endarterectomy operation, have advanced lesion type (fibrocalcific), which are composed of mainly two regions; collagen-rich and calcific. Few TFCAs, which are hard to come across during these operations, are examined in our study, since microcalcifications within TCFAs, are the indicators of plaque rupture.

Sound speed and acoustic impedance modes are the two modes of SAM. More widely, in acoustic property investigation studies of tissues or cells, sound speed mode has been used. In this mode, there is a requirement of very thin samples, which necessitates the use of a microtome. However, in acoustic impedance mode, no special sampling is necessary and therefore, that mode is more straightforward. Acoustic microscopy in sound speed mode demonstrated different speed of sound values due to variation of elasticity within the atherosclerotic coronary arteries [18, 19]. Similarly, carotid atherosclerotic plaques were characterized by measurement of sound speed ex vivo and different plaque components were observed to have different values of sound speed [20].

In our study, acoustic impedance in the atherosclerotic plaques was measured with SAM. In acoustic impedance mode, tissues are not prepared as thin slices. The samples with flat surfaces in contact with the substrate, is the only requirement. The results show that the acoustic impedance value of the lipid pool has the lowest value, since the elasticity of lipids is low. Higher values of acoustic impedance for the fibrous tissue are expected, since, fibrous tissue has higher elasticity compared to lipids. The variation of acoustic impedance values within the fibrous tissue is smaller than the variation in the lipid pool, since, the ratio of the lipid pool is not always significant in plaque formation, instead, fibrous tissue is significant. In the calcified lesion, the elasticity is even higher, therefore the acoustic impedance was observed to be the highest. The larger standard deviation in calcified region results from rupture into lumen and expose calcium nodules, which become sites for thrombosis. The assessment of plaque vulnerability is possible by providing morphological and together with chemical information about the plaques, which can be obtained by SAM.

the fact that calcium deposition was low in the plaques, which are TCFAs, investigated.

\section{Conclusion}

The acoustic properties of atherosclerotic plaques studied by scanning acoustic microscopy showed different acoustic properties due to elasticity variation within the TCFA samples. We can conclude that the success of SAM in determining calcifications at the microscopic level is a very important advantage, since it can determine vulnerability of plaques with a very quick scan of the arteries and therefore, will help in decision making of the surgeries, if in vivo implementation of $\mathrm{SAM}$ is achieved in the future.

\section{Acknowledgement}

This study is supported by Ministry of Development of Turkey. Project Number: 2009K120520.

\section{References}

1. Bentzon, JF, Otsuka, F, Virmani, R, Falk, E, Mechanism of Plaque formation and Rupture, Circulation Research, 2014, 114, 1852-1866.

2. Insull, W, The Pathology of Atherosclerosis: Plaque Development and Plaque Responses to Medical Treatment, The American Journal of Medicine, 2009, 122(1A), S3-14.

3. Puri, R, Worthley, MI, Nicholls, SJ, Intravascular imaging of vulnerable coronary plaque: current and future concepts, Nature Reviews Cardiology, 2011, 8, 131-139.

4. Carlier, SG, Tanaka, K, Studying Coronary Plaque Regression with IVUS: A Critical Review of Recent Studies, Journal of Interventional Cardiology, 2016, 19(1), 11-15.

5. Li, J, Chen, Z, Integrated intravascular ultrasound and optical coherence tomography technology: a promising tool to identify vulnerable plaques, Journal of Biomedical Photonics and Engineering, 2016, 1(4), 209-224.

6. Jansen, K, Van Soest, G, Van Der Steen, AF, Intravascular photoacoustic imaging: a new tool for vulnerable plaque identification, Ultrasound in Medicine and Biology, 2014, 40(6), 1037-1048.

7. Wang, B, Su, JL, Karpiouk, AB, Intravascular Photoacoustic Imaging, IEEE Journal of Selected Topics in Quantum Electronics, 2010, 16(3), 599-599.

8. Yeager, D, Karpiouk, A, Wang, B, Amirian, J, Sokolov, K, Smalling, R, Emelianov, S, Intravascular photoacoustic imaging of exogenously labeled atherosclerotic plaque through luminal blood, Journal of Biomedical Optics, 2012, 17(10), 106016.

9. Berer, T, Grun, H, Bauer-Marschallinger, J, Burgholzer, P, Passler, K Nuster, R, Paltauf, G. In IEEE International Ultrasonics Symposium, Dresden, Germany, 2012, 10.1109/ULTSYM.2012.0356.

10. Kobayashi, K, Yoshida, S, Saijo, Y, Hozumi, N, Acoustic impedance microscopy for biological tissue characterization, Ultrasonics, 2014, 54, 1922-1928. 
11. Hozumi, N, Nakano, A, Terauchi, S, Nagao, M, Yoshida, S, Kobayashil, , Shanahan, CM, Inflammation Ushers in Calcification A Cycle of Damage Yamamoto, S, Saijo, Y. In IEEE International Ultrasonics Symposium,and Protection?, Circulation, 2007, 116, 2782-2785. New York, NY, USA, 2007, 1051-0117/07.

17. Aikawa, E, Nahrendorf, M, Figueiredo, JL, Swirski, FK, Shtatland, T,

12. De Korte, DL, Pasterkamp, G, Van Der Steen, AFW, Woutman, HA, Bom,Kohler, RH, Farouc, AJ, Aikawa, M, Weissleder, R, Osteogenesis $\mathrm{N}$, Characterization of Plaque Components with Intravascular UltrasoundAssociates With Inflammation in Early-Stage Atherosclerosis Evaluated Elastography in Human Femoral and Coronory Arteries In Vitro,by Molecular Imaging In Vivo, Circulation, 2007, 116, $2841-2850$. Circulation, 2000, 102(6), 617-623.

18. Saijo, Y, Ohashi, T, Sasaki, H, Sato, M, Jorgensen, CS, Nitta, S, . Virmani, R, Kolodgie, FD, Burke, AP, Farb A, Schwartz, SM, Lessons Application of Scanning Acoustic Microscopy for Assessing Stress from sudden coronary death: a comprehensive morphologicalDistribution in Atherosclerotic Plaque, Annals of Biomedical Engineering, classification scheme for atherosclerotic lesions, Arteriosclerosis,2001, 29, 1048-1053.

Thrombosis, and Vascular Biology, 2000, 20, 1262-1275.

19. Saijo, Y, Hozumi, N, Lee, C, Nagao, M, Kazuto, K, Oakada, N, Filho, ES, Calcification: Translating Biology, Current Atherosclerosis Reports, 2016,of coronary artery, Ultrasonics, 2006, 44, e51-e55.

18,51 .

20. Brewin, MP, Srodon, PD, Greenwald, SE, Birch, MJ, Carotid

15. Vengrenyuk, Y, Carlier, S, Xanthos, S, Cardoso, L, Ganatos, $\mathrm{P}$, Virmani,atherosclerotic plaque characterization by measurement of ultrasound R, Einav, S, Gilchrist, L, Weinbaum, S, A hypothesis for vulnerablesound speed in vitro at high frequency, $20 \mathrm{MHz}$, Ultrasonics, 2014, 54, plaque rupture due to stress-induced debonding around cellular428-441.

microcalcifications in thin fibrous caps, Proceedings of the National

Academy of Sciences of the United States of America, 2006, 103, 14678-

14683 . 